\title{
Implementación electrónica del oscilador caótico de Rössler
}

\section{Electronic implementation of the Rössler chaotic oscillator}

\author{
SILVA-JUÁREZ, Alejandro†*, ESTEVEZ-CARREÓN, Jaime, PONCE-MELLADO, Juan Jorge y \\ HERRERA-SÁNCHEZ, Gustavo
}

Universidad Tecnológica de Puebla, México.

Instituto Tecnológico de Puebla, México.

ID $1^{\mathrm{er}}$ Autor: Alejandro, Silva-Juárez / ORC ID: 0000-0001-8473-9803, Researcher ID Thomson: F-6969-2018, arXiv ID Author: Alejandro_Silva, CVU CONACYT ID: 637028

ID $1^{\text {er }}$ Coautor: Jaime, Estevez-Carreón / ORC ID: 0000-0002-2549-3345, Researcher ID Thomson: N-7519-2013, CVU CONACYT ID: 200706

ID $2^{\text {do }}$ Coautor: Juan Jorge, Ponce-Mellado / ORC ID: 0000-0003-2186-2868, Researcher ID Thomson: G-6040-2018, arXiv ID Author: Jorge_Ponce, CVU CONACYT ID: 900658

ID $3{ }^{\text {er }}$ Coautor: Gustavo, Herrera-Sánchez / ORC ID: 0000-0001-5276-5062, Researcher ID Thomson: F-6595-2018, arXiv ID Author: herreragh, CVU CONACYT ID: 459805

DOI: $10.35429 / J C P E .2020 .22 .7 .18 .25$

Recibido 25 de Enero, 2020; Aceptado 30 de Junio, 2020

Resumen

Los sistemas caóticos han llamado la atención de la comunidad científica debido a sus potenciales aplicaciones en muchas ramas de las ciencias e ingeniería, la teoría del caos se utiliza en la criptografía, en la creación de modelos poblacionales en biología, estudiando la turbulencia en la mecánica de fluidos, en economía se utiliza para predecir el comportamiento del mercado de valores, en astronomía para mejorar la predicción de trayectorias en asteroides y determinar si entran o no en contacto con la tierra, en años más recientes se ha aplicado en la predicción y/o control de la dinámica del cerebro humano, se han reportado un gran número de trabajos en diferentes áreas con el fin de abordar algunos problemas interesantes, como la determinación de daños estructurales en materiales $o$ en sistemas de comunicaciones seguras. En este trabajo se aborda la implementación de osciladores caóticos en electrónica analógica, se presenta el caso de estudio del sistema dinámico no lineal autónomo de Rössler, se desarrolla el análisis de estabilidad del sistema, se incluyen resultados analíticos, numéricos y experimentales, así como su implementación electrónica medida en condiciones de laboratorio para observar atractores caóticos experimentales, los cuales serán utilizados en la implementación de generadores de números aleatorios y sistemas de comunicación segura para el cifrado de imágenes.

Caos, Osciladores, Implementación Analógica

\begin{abstract}
Chaotic systems have attracted the attention of the scientific community due to their potential applications in many branches of science and engineering, chaos theory is used in cryptography, in the creation of population models in biology, studying turbulence in mechanics of fluids, in economics it is used to predict the behavior of the stock market, in astronomy it is used to describe the motion of many planetary bodies, to improve the prediction of trajectories in asteroids and to determine whether or not they come into contact with the earth, in More recent years have been applied in the prediction and / or control of the dynamics of the human brain, a large number of works have been reported in different areas in order to address some interesting problems, such as the determination of structural damage in materials or in secure communication systems. In this work the implementation of chaotic oscillators in analog electronics is addressed, the case study of the autonomous non-linear dynamic system of Rössler is presented, the stability analysis of the system is developed, analytical, numerical and experimental results are included, as well as its Electronic implementation measured under laboratory conditions to observe experimental chaotic attractors, which will be used in the implementation of random number generators and secure communication systems for image encryption.
\end{abstract}

Chaos, Oscillators, Analog Implementation

Citación: SILVA-JUÁREZ, Alejandro, ESTEVEZ-CARREÓN, Jaime, PONCE-MELLADO, Juan Jorge y HERRERASÁNCHEZ, Gustavo. Implementación electrónica del oscilador caótico de Rössler. Revista de Energía Química y Física. 2020. 7-22:18-25.

\footnotetext{
* Correspondencia al Autor (Correo Electrónico: alejandro.silva@utpuebla.edu.mx)

$\dagger$ Investigador contribuyendo como primer autor
} 


\section{Introducción}

El estudio de los sistemas dinámicos no lineales y el caos se ha convertido en un tema de gran interés y atrajo un enorme interés de investigación después de la demostración numérica del caos por Lorenz (1963). Los sistemas caóticos están asociados con comportamientos dinámicos complejos que poseen algunas características especiales que incluyen trayectorias limitadas con exponentes positivos de Lyapunov y dependencia sensible de las condiciones iniciales.

El estudio de la dinámica caótica ha encontrado aplicación en varios campos de las disciplinas científicas y de ingeniería, incluyendo meteorología, física, química, ingeniería, economía, biología, comunicación segura, cifrado y procesamiento de control [1-3].

Los sistemas caóticos se conocen desde hace mucho tiempo, pero solo recientemente se demostró que el caos podía controlarse y, por lo tanto, sincronizarse [4-6]. Por esta razón; Esta clase de sistemas promete tener un gran impacto en muchas aplicaciones novedosas, críticas en términos de tiempo y energía, tales como circuitos electrónicos y dispositivos de alto rendimiento, el procesamiento seguro de la información [7-10].

Esta nueva y desafiante área de investigación y desarrollo se ha convertido en una interdisciplina científica, que involucra ingenieros de sistemas y control, físicos teóricos y experimentales, matemáticos aplicados, fisiólogos $\mathrm{y}$, sobre todo, especialistas en circuitos y dispositivos.

Hoy en día, los generadores de caos han recibido considerable atención para modelarlos, simularlos, diseñarlos y usarlos en aplicaciones de ingeniería. El interés está en los problemas teóricos y prácticos, mientras que una aplicación directa está en el campo de la sincronización caótica para implementar esquemas de comunicación privada, donde la información confidencial que se transmite se incrusta en una señal caótica mediante modulación directa, enmascaramiento u otra técnica $[11,12]$. En el extremo del receptor, si la sincronización caótica se logra perfectamente, se puede extraer la información oculta de la señal transmitida.
En este trabajo se realiza el análisis de estabilidad del sistema de Rössler, las aproximaciones, simulaciones para su implementación con dispositivos analógicos como amplificadores operacionales y arreglos R-C para utilizarlo en aplicaciones de comunicación segura en transmisión de imágenes.

\section{Sistemas dinámicos no lineales autónomos}

Uno de los sistemas de ecuaciones diferenciales no lineales que presenta un comportamiento caotico es el sistema de Rössler [13, 14], las ecuaciones de variables de estado del sistema se muestran en (1).

$$
\begin{aligned}
& \frac{d x}{d t}=-y-z \\
& \frac{d y}{d t}=x+a y \\
& \frac{d z}{d t}=b x-c z+x z
\end{aligned}
$$

En la ecuacion (1) $(x, y, z) \in \mathbb{R}^{3}$ son las tres variables dinámicas que evolucionan en el tiempo continuo $t$ que definen el espacio de fase y $(a, b, c) \in \mathbb{R}^{3}$ son sus parámetros: $a=$ $0.2 ; b=0.2 ; c=7.0$, para el análisis de estabilidad de los sistemas caoticos se lleva a cabo la siguiente metodología:

- Obtener los puntos de equilibrio.

- Aplicar la matriz jacobiana.

- Calcular los valores propios.

- Determinar la estabilidad del sistema.

- Determinar el tamaño de paso h.

- Resolver el sistema con el método numérico.

- Calcular las métricas: Exponente de Lyapunov, entropía, dimensión fractal.

Para encontrar los puntos de equilibrio, las tres ecuaciones de Rössler (1) se establecen en cero y las coordenadas $(x, y, z)$ de cada punto de equilibrio se determinan resolviendo las ecuaciones resultantes. 
Esto produce las ecuaciones generales de cada una de las coordenadas de punto de equilibro. El sistema de Rössler tiene dos puntos de equilibrio $E_{1,2}$ situados en:

$$
\begin{aligned}
& E_{1}=\left(\frac{c+\sqrt{c^{2}-4 a b}}{2},-\frac{-c-\sqrt{c^{2}-4 a b}}{2 a}, \frac{c+\sqrt{c^{2}-4 a b}}{2 a}\right) \\
& E_{2}=\left(\frac{c-\sqrt{c^{2}-4 a b}}{2},-\frac{-c+\sqrt{c^{2}-4 a b}}{2 a}, \frac{c-\sqrt{c^{2}-4 a b}}{2 a}\right)
\end{aligned}
$$

La estabilidad de cada uno de los puntos de equilibrio $E_{1,2}$ son analizados determinando sus respectivos valores propios, de este modo, la matriz jacobiana del sistema (1) en los puntos de equilibrio $E_{1} y E_{2}$ esta dada por:

$$
J=\left(\begin{array}{lll}
\frac{\partial f_{1}}{\partial x} & \frac{\partial f_{1}}{\partial y} & \frac{\partial f_{1}}{\partial z} \\
\frac{\partial f_{2}}{\partial x} & \frac{\partial f_{2}}{\partial y} & \frac{\partial f_{2}}{\partial z} \\
\frac{\partial f_{3}}{\partial x} & \frac{\partial f_{3}}{\partial y} & \frac{\partial f_{3}}{\partial z}
\end{array}\right)=\left[\begin{array}{ccc}
0 & -1 & -1 \\
1 & a & 0 \\
z & 0 & x-c
\end{array}\right]
$$

La ecuacion característica tiene la forma $|\lambda I-J|=0$, aplicando se obtienen los valores propios del sistema (1) y resolviendo en los puntos de equilibrio se obtiene la ecuación cubica:

$$
-\lambda^{3}+\lambda^{2}(a+x-c)+\lambda(a c-a x-1-z)+x-c+a z=0
$$

Para el punto de equilibrio $E_{1}$ con parámetros $\quad a=0.2 ; b=0.2 ; c=7.0 \quad$ se obtienen los siguientes valores propios: $\lambda_{1} \approx$ $0.47595, \lambda_{2,3} \approx 0.007017 \pm 4.57910 i$. Para el punto de equilibrio $E_{2}$ se obtienen los siguientes valores propios: $\lambda_{1} \approx-9.98800$, $\lambda_{2,3} \approx 0.249007 \pm 0.96808 i$. De acuerdo con los resultados de los valores propios, el punto de equilibrio $E_{1}$ se encuentra localizado en el centro del atractor y se denomina un foco-silla inestable en el plano $x-y$, el punto de equilibrio $E_{2}$ es un foco silla también de tipo inestable, las cuales son características que aseguran un comportamiento caótico.

En los Gráficos 1 a 4 se muestran los resultados de las simulaciones numéricas del sistema de Rössler, en las cuales se visualiza la creación de un oscilador caótico que es sensible a las condiciones iniciales, para esto se aplica el método de integración de Rünge-Kutta de cuarto orden con $25 \times 10^{3}$ iteraciones [15].
Cada aproximación numérica se ha realizado con tamaño de paso de integración $h=0.001$, tiempo de simulación $t_{\text {sim }}=200$ segundos $y$ condiciones iniciales $(x(0), y(0), z(0))=(0.5,1.5,0.1), \quad$ los algoritmos se programaron usando el modelo matemático (1), los parámetros $a=0.2 ; b=$ $0.2 ; c=7.0$ y Matlab R2020a ${ }^{\circledR}$ en doble precisión, el tipo de error por redondeo y épsilon de máquina de 2.2204e-16. En el Gráfico 5 se muestran las series de tiempo de las variables $x, y, z$, donde se muestra una de las características más importantes de sistemas caóticos y que es la sensibilidad a las condiciones iniciales, ya que conforme transcurre el tiempo las trayectorias se separan de forma exponencial.

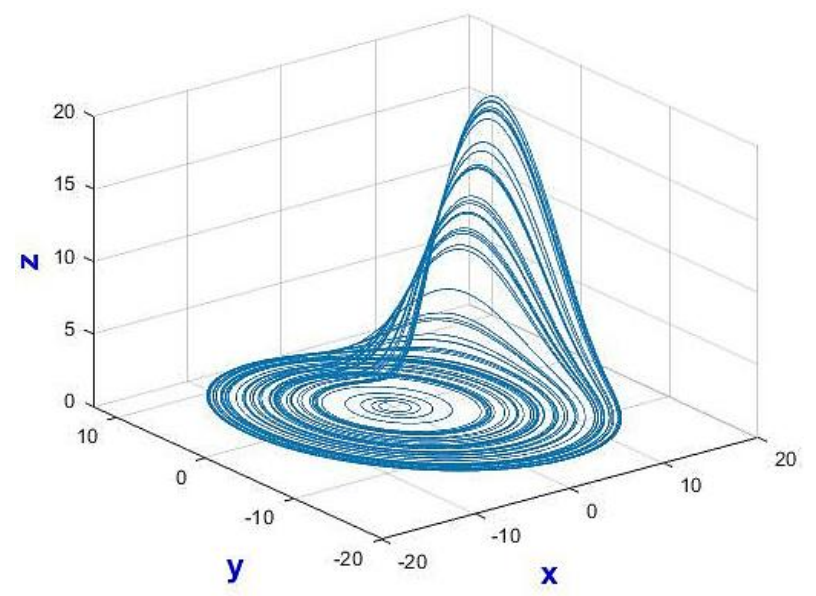

Gráfico 1 Resultado de la simulación numérica del sistema de Rössler en espacio de estados en el plano 3D

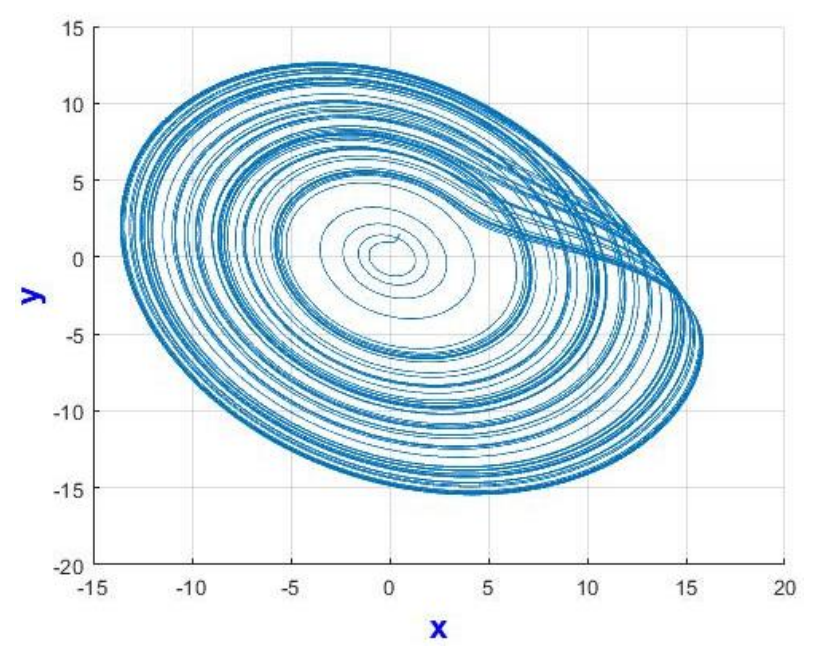

Gráfico 2 Resultado de la simulación numérica del sistema de Rössler en espacio de estados en el plano xy 


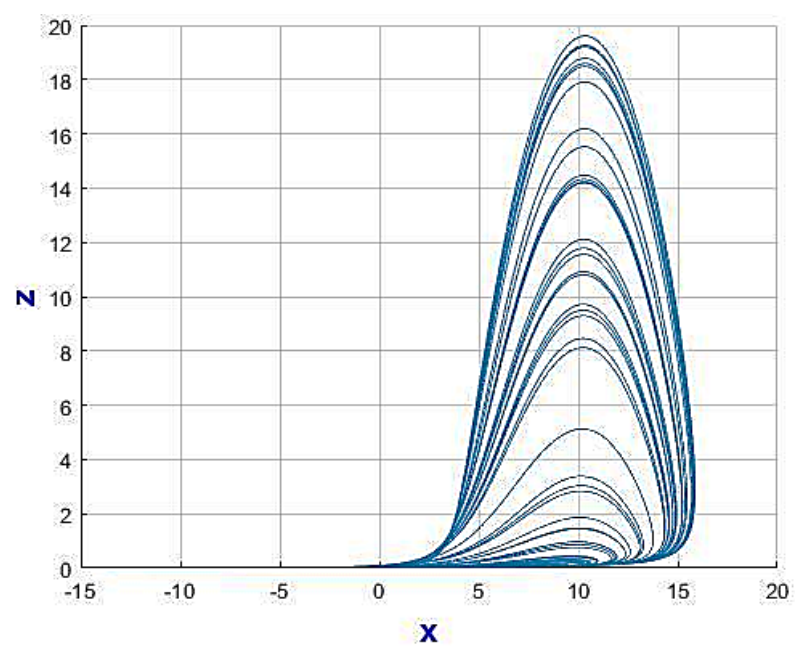

Gráfico 3 Resultado de la simulación numérica del sistema de Rössler en espacio de estados en el plan xz

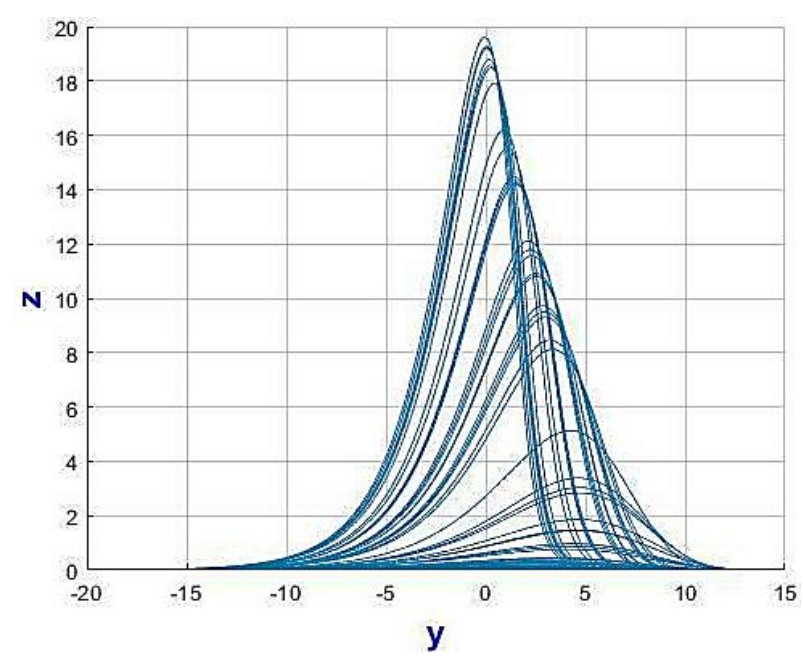

Gráfico 4 Resultado de la simulación numérica del sistema de Rössler en espacio de estados en el plano yz

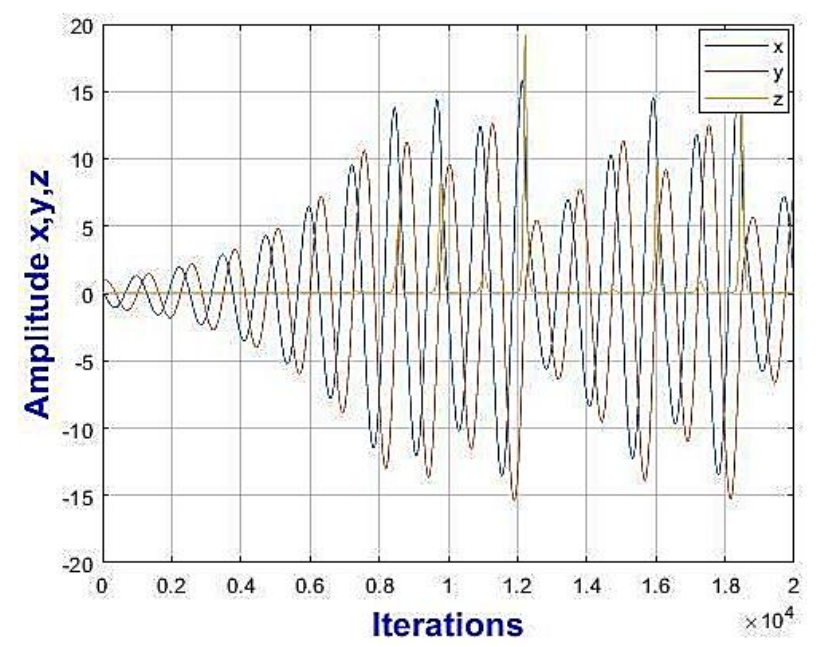

Gráfico 5 Simulaciones numéricas de la ecuación (1): tendencia de las variables de estado $\mathrm{x}, \mathrm{y}, \mathrm{z}$

\section{Implementación analógica}

La realización electrónica del sistema caótico de Rössler puede realizarse con amplificadores operacionales, elementos pasivos $\mathrm{R}-\mathrm{C}$ y un multiplicador analógico, el procedimiento de diseño comienza analizando en comportamiento dinámico de las series de tiempo de las variables $x, y$ y $z$ del sistema (1) como se observa en la figura $6(\mathrm{a}, \mathrm{b}, \mathrm{c})$. Es importante considerar para efectos de implementación, que la dinámica de las tres variables de estado presenta rangos de amplitudes $(-21.5 ; 21.5)$ para $x,(-15.4 ; 16.8)$ para $y$ y $(-0 ; 22)$ para $z$, es importante notar que estos rangos exceden los valores de salida de voltaje de los amplificadores operacionales que comúnmente son $\pm 12 \mathrm{~V}$.

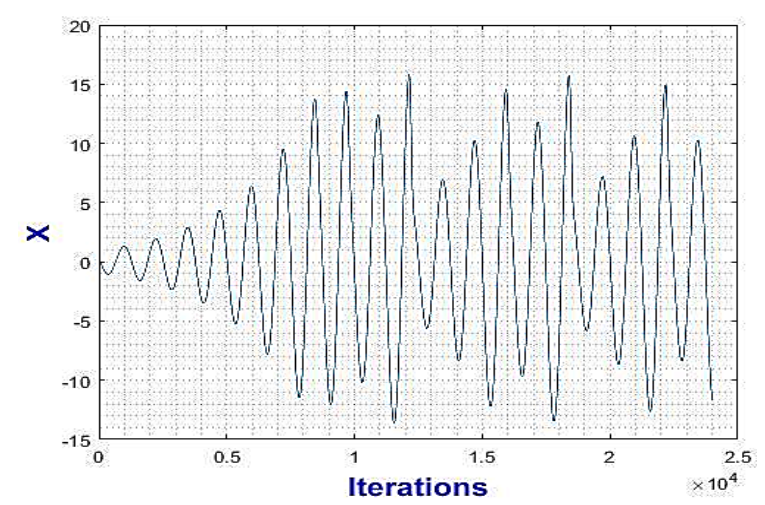

a)

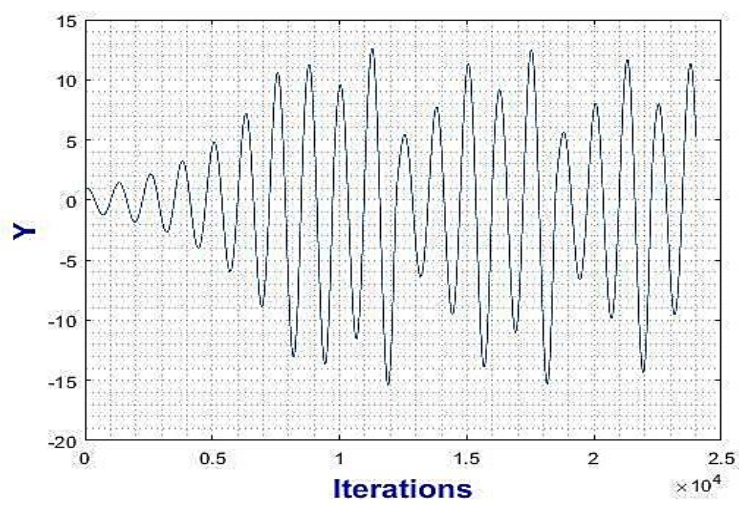

b)

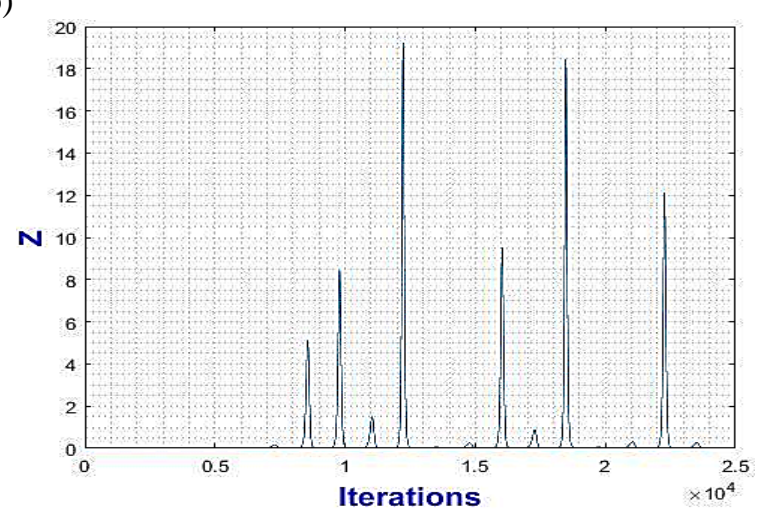

c)

Gráfico 6 Series de tiempo vs amplitudes de las variables de estado del atractor de Rössler: a) x, b) y c) z. 
Una implementación del sistema (1) se muestra en las Figuras 1, 2, 3 y 4, el circuito analógico requiere de tres amplificadores operacionales que pueden ser LM741 o TL082, donde, cada uno realiza la operación de sumador, restador e integrador, y un multiplicador analógico AD633AN para implementar el término no lineal en las ecuaciones del circuito formado por el producto de las variables de estado $x z$. Implementar directamente el sistema de ecuaciones de (1) en su forma original en un circuito analógico implica un escalamiento, ya que las variables de estado ocupan un rango dinámico con valores que exceden los límites del voltaje de alimentación típica de un OPAMP $\pm 12 \mathrm{~V}$ como se puede observar en el Gráfico 2. Las elecciones de diseño llevan a elegir los factores de escala de la siguiente manera:

$X=k_{1} x ; \quad Y=k_{2} y ; Z=k_{3} Z$

Donde:

$k_{1}=\frac{1}{10} ; k_{2}=\frac{1}{10} ; k_{3}=\frac{1}{10}$

De esta forma se encuentra un nuevo sistema equivalente reescalado:

$$
\left\{\begin{array}{c}
\dot{X}=-Y-Z \\
\dot{Y}=X+a Y \\
\dot{Z}=\frac{b}{10}-10 X Z-c Z
\end{array}\right.
$$

El nuevo sistema reescalado ahora se simula y se verifica que las amplitudes de las variables de estado oscilan dentro de los límites de voltaje que ahora son realizables. De esta forma, se verifica la viabilidad del circuito. La implementación de la primera ecuación reescalada $\dot{x}=-Y-Z$ necesita un integrador R-C y un sumador, con el objetivo de utilizar el integrador $\mathrm{R}-\mathrm{C}$, la ecuación se reescribe en la forma $\dot{x}=-X+X-Y-Z$. Partiendo de esta ecuación se obtiene el siguiente circuito:

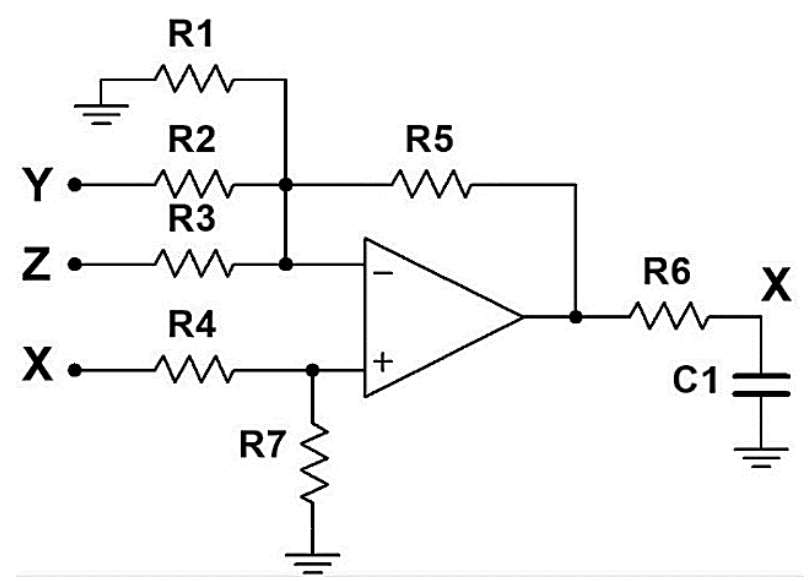

Figura 1 Circuito asociado a la variable $\mathrm{x}$ del sistema (1). Los parámetros son: $\mathrm{R}_{1}=\mathrm{R}_{2}=\mathrm{R}_{3}=\mathrm{R}_{4}=\mathrm{R}_{5}=100 \mathrm{k} \Omega, \mathrm{R}_{6}$ $=1 \mathrm{k} \Omega, \mathrm{R}_{7}=33.3 \mathrm{k} \Omega$ y $\mathrm{C}_{1}=100 \mathrm{nF}$

La elección de $C_{1}$ y $R_{6}$ en la figura 7 corrige el cambio de escala de tiempo como $k=$ $1 /\left(R_{6} C_{1}\right)=1000$, entonces el circuito tiene la siguiente ecuación:

$C_{1} R_{6} \dot{X}=-X+\frac{R_{5}}{R_{4}} X-\frac{R_{5}}{R_{2}} Y-\frac{R_{5}}{R_{3}} Z$

De forma similar, se procede con las otras dos variables la segunda ecuación reescalada es reescrita como: $\dot{Y}=-Y+X+(a+1) Y$. El esquema del circuito es reportado en la figura 8 y la ecuación toma la siguiente forma:

$C_{2} R_{12} \dot{Y}=-Y+\frac{R_{11}}{R_{9}} X-\frac{R_{11}}{R_{10}} Y$

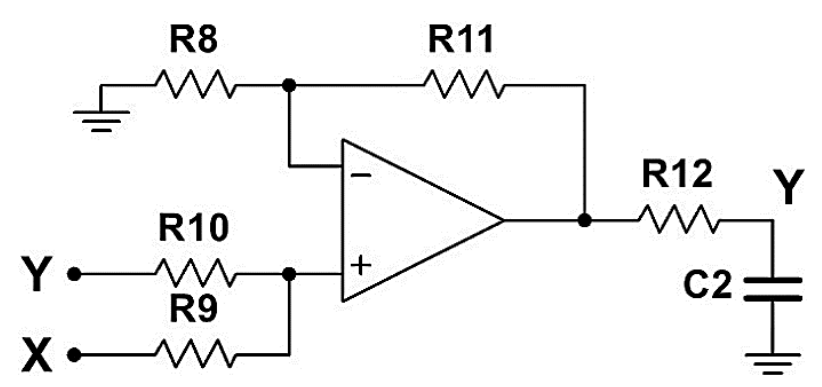

Figura 2 Circuito asociado a la variable y del sistema (1). Los parámetros son: $\mathrm{R}_{8}=\mathrm{R}_{10}=78 \mathrm{k} \Omega, \mathrm{R}_{9}=\mathrm{R}_{11}=100 \mathrm{k} \Omega$, $\mathrm{R}_{12}=1 \mathrm{k} \Omega$ y $\mathrm{C}_{2}=100 \mathrm{nF}$

Finalmente, el tercer bloque de la ecuacion reescalada para la variable $Z$ se reescribe como:

$\dot{Z}=-Z+\frac{b}{10}+10 X Z+Z(1-c)$ 
En esta ecuación aparecen dos nuevos tipos de términos, un término constante $b / 10$ y un término no lineal $X Y$. Para implementar el primer término se usa un divisor de voltaje, el segundo término es realizado usando un multiplicador analógico AD633AN como se muestra en la figura 3 , tomando en cuenta que la salida del multiplicador está dada por:

$W=\frac{\left(X_{1}-X_{2}\right)\left(Y_{1}-Y_{2}\right)}{10 \mathrm{~V}} \frac{R_{22}+R_{23}}{R_{22}}$

De modo que, si se selecciona $X 1=$ $X, X 2=0, Y 1=Y, Y 2=0 \quad$ y la relación $\frac{R_{22}+R_{23}}{R_{22}}=10$, se obtiene el término que proporciona la no linealidad al sistema caotico de Rössler al multiplicar las variables $X Y$ en la tercera ecuacion del sistema (1).

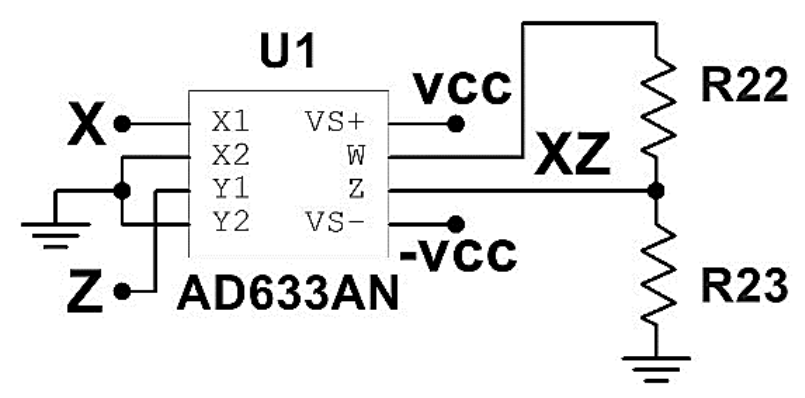

Figura 3 Circuito multiplicador de las variables $X Y$ en la tercera ecuacion del sistema (1). Los parámetros son: $\mathrm{R}_{22}$ $=1 \mathrm{k} \Omega, \mathrm{R}_{23}=9 \mathrm{k} \Omega$.

La tercera ecuación del sistema de Rossler para la variable $z$ tiene entonces la siguiente forma:

$$
C_{3} R_{21} \dot{Z}=-Z+\frac{R_{18} R_{25}}{R_{17} R_{24}} Z+\frac{R_{18}}{R_{16}} X Z+\frac{R_{18}}{R_{15}} \frac{R_{20}}{R_{19}+R_{20}} \mathrm{~V}_{\mathrm{cc}}
$$

Y su circuito se muestra en la figura 10.

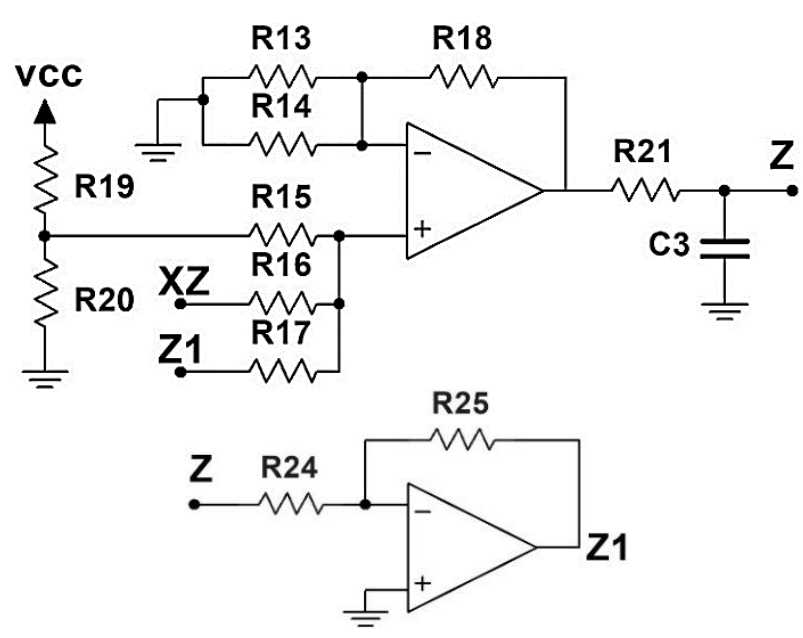

Figura 4 Circuito asociado a la variable $\mathrm{z}$ del sistema (1). Los parámetros son: $\mathrm{R}_{13}=\mathrm{R}_{15}=\mathrm{R}_{17}=\mathrm{R}_{18}=100 \mathrm{k} \Omega, \mathrm{R}_{14}=$ $\mathrm{R}_{16}=\mathrm{R}_{24}=10 \mathrm{k} \Omega, \mathrm{R}_{19}=449 \mathrm{k} \Omega, \mathrm{R}_{20}=\mathrm{R}_{21}=1 \mathrm{k} \Omega, \mathrm{R}_{25}=$ $80 \mathrm{k} \Omega$ (potenciómetro) y $\mathrm{C}_{3}=100 \mathrm{nF}$
Se hace referencia que $C_{1} R_{6}=C_{2} R_{12}=$ $C_{3} R_{21}$ para que la escala de tiempo sea coherente para todas las ecuaciones del sistema. Una vez diseñado el esquema del circuito de Rössler, se verifica a través de la herramienta de simulación Multisim 14.2 de National Instruments. Construyendo los circuitos de las figuras 7 a 10 y uniendo las etiquetas de las variables, se obtiene la implementación del circuito analógico del sistema de Rössler, los resultados de la simulación en multisim muestran la creación del atractor en las figuras 5 a 7 .

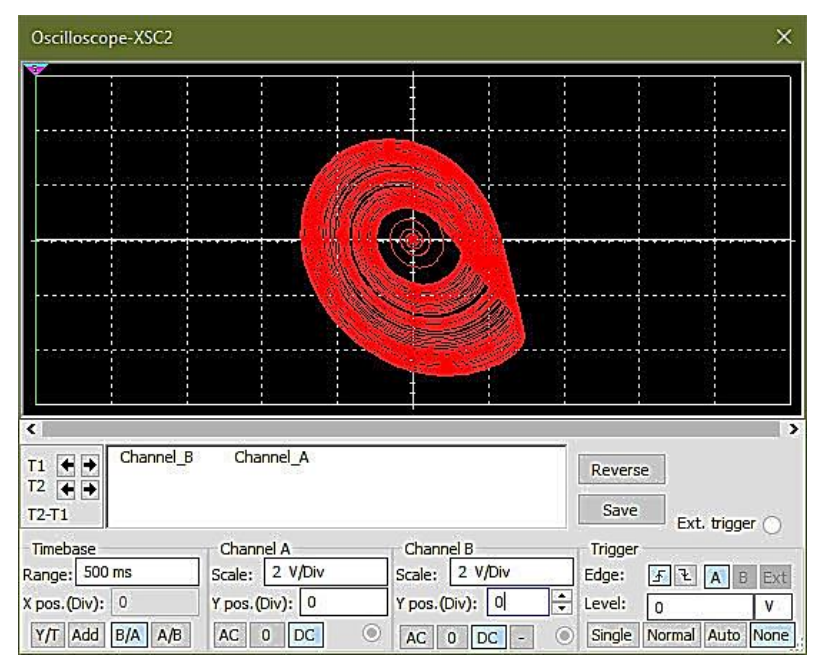

Figura 5 Atractor caótico del sistema de Rössler (2V/Div): plano xy

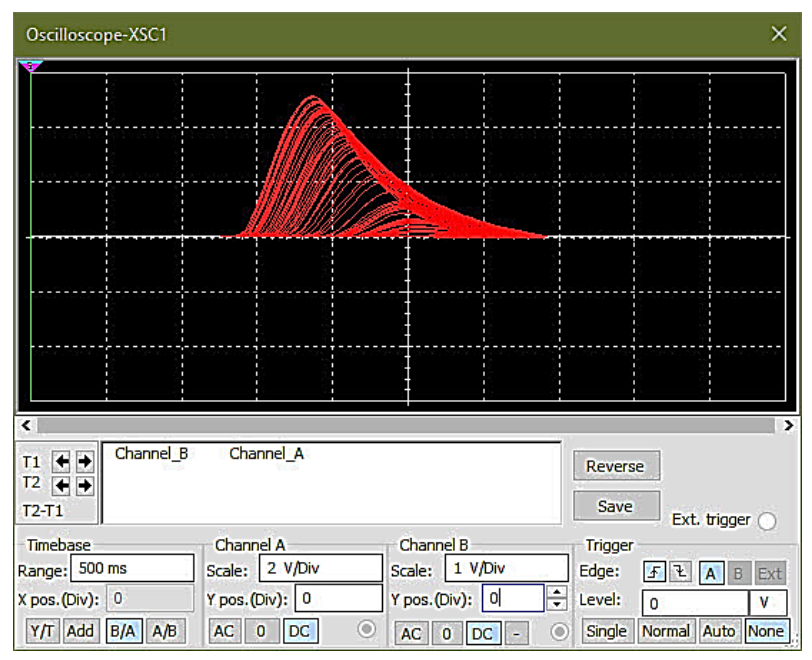

Figura 6 Atractor caótico del sistema de Rössler (2V/Div): plano $\mathrm{xz}$

SILVA-JUÁREZ, Alejandro, ESTEVEZ-CARREÓN, Jaime, PONCE-MELLADO, Juan Jorge y HERRERA-SÁNCHEZ, Gustavo. Implementación electrónica del oscilador caótico de Rössler. Revista de Energía Química y Física. 2020 


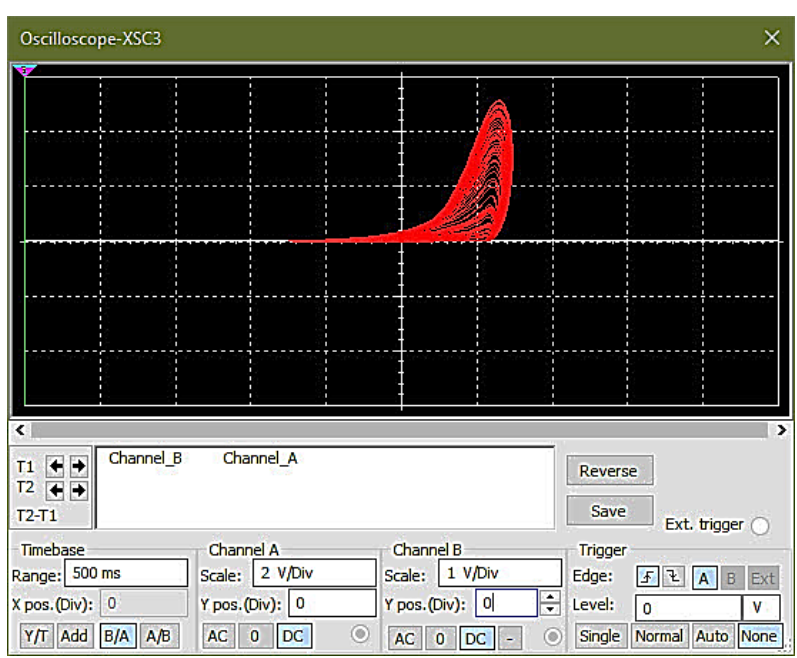

Figura 7 Atractor caótico del sistema de Rössler (2V/Div): plano yz

\section{Conclusiones}

Se ha presentado la implementación electrónica analógica basada en OPAMPs del sistema caótico de Rössler, que es adecuado para un diseño reconfigurable de acuerdo con su dinámica autónoma no lineal, se presenta la metodología de análisis de estabilidad, simulación de las aproximaciones numéricas y se describe el diseño de los circuitos de las tres variables de estado del sistema. Los resultados experimentales concuerdan con los resultados obtenidos de experimentos establecidos por componentes discretos como los FPGA y los FPAA debido a que el sistema ha sido reescalado y los voltajes de salida del oscilador son adecuados para esta tecnología y fácilmente adaptados. Este sistema puede usarse efectivamente como un generador de caos programable en muchas aplicaciones basadas en el caos, como la sincronización de sistemas maestro-esclavo para transmisión de imagen, criptografía y seguridad en comunicaciones.

\section{Referencias}

[1] Gaspard, P.: Encyclopedia of Nonlinear Science. In: "Rössler Systems", pp. 808811. Routledge (2005).

[2] T. Parker and L. Chua, "The dual double scroll equation," IEEE Transactions on Circuits and Systems, vol. CAS-34, no. 9, pp. 1059-1073, 1987.

[3] L. M. Pecora and T. L. Carroll, "Synchronization in chaotic systems," Physical review letters, vol. 64, no. 8, p. 821, 1990.
[4] K. M. Cuomo and A. V. Oppenheim, "Circuit implementation of synchronized chaos with applications to communications," Physical review letters, vol. 71, no. 1, p. 65, 1993.

[5] S. Yu, Z. Ma, S. Qiu, S. Peng, and Q. Lin, "Generation and synchronization of nscroll chaotic and hyperchaotic attractors in fourthorder systems," Chin. Phys., no. 13, pp. 317-328, 2004.

[6] P. Stavroulakis, Chaos applications in telecommunications. CRC press, 2005.

[7] Kapitaniak, Chaos for engineers: theory, applications, and control. Springer Science \& Business Media, 2012.

[8] Silva-Juarez, A., Rodriguez-Gomez, G., de la Fraga, L. G., Guillen-Fernandez, O., \& Tlelo-Cuautle, E. (2019). Optimizing the kaplan-yorke dimension of chaotic oscillators applying de and pso. Technologies, 7(2), 38.

[9] Tlelo-Cuautle, E., Pano-Azucena, A. D., Guillén-Fernández, O., \& Silva-Juárez, A. (2020). Integer and Fractional-Order Chaotic Circuits and Systems. In Analog/Digital Implementation of Fractional Order Chaotic Circuits and Applications (pp. 1-40). Springer, Cham.

[10] Silva-Juárez, A., Morales-Pérez, C. J., de la Fraga, L. G., Tlelo-Cuautle, E., \& de Jesús Rangel-Magdaleno, J. (2019). On maximizing the positive Lyapunov exponent of chaotic oscillators applying $\mathrm{DE}$ and PSO. International Journal of Dynamics and Control, 7(4), 1157-1172.

[11] H. Degn, A. V. Holden, and L. F. Olsen, Chaos in biological systems. Springer Science \& Business Media, 2013, vol. 138.

[12] R. Weller, "Resistance, chaos and control in china: Taiping rebels, taiwanese ghosts and tiananmen," 2013.

[13] S. H. Strogatz, Nonlinear dynamics and chaos: with applications to physics, biology, chemistry, and engineering. Westview press, 2014. 
[14] Curry, J. H. (1978). A generalized Rössler system. Communications in Mathematical Physics, 60(3), 193-204.

[15] Chapra, S. C., \& Canale, R. P. (2010). Numerical methods for engineers. Boston: McGraw-Hill Higher Education. 